\title{
NEW BOUNDS FOR IRRATIONALITY MEASURES OF SOME FAST CONVERGING SERIES
}

\author{
JAN ŠUSTEK
}

\begin{abstract}
This paper presents new upper bounds for irrationality measures of some fast converging series of rational numbers. The results depend only on the speed of convergence of the series and do not depend on the arithmetical properties of the terms.
\end{abstract}

\section{INTRODUCTION}

For a real number $\xi$, its irrationality measure $\mu(\xi)$ is defined as the supremum of all positive real numbers $\mu$ such that the inequality

$$
0<\left|\xi-\frac{p}{q}\right|<\frac{1}{q^{\mu}}
$$

has infinitely many solutions $p \in \mathbb{Z}, q \in \mathbb{Z}^{+}$. Irrationality measure describes how closely the number $\xi$ can be approximated by rational numbers. All irrational numbers $\xi$ have irrationality measure $\mu(\xi) \geq 2$. A famous result of Roth [5] is that all algebraic irrational numbers $\xi$ have irrationality measure $\mu(\xi)=2$. Sondow [6] showed that if $\frac{p_{n}}{q_{n}}$ are the convergents of the continued fraction of a number $\xi$ then

$$
\mu(\xi)=1+\limsup _{n \rightarrow \infty} \frac{\log q_{n+1}}{\log q_{n}} .
$$

Adamczewski and Rivoal [1] found an upper bound for irrationality measure of a number $\xi$ depending on the growth properties of rational approximants of $\xi$.

Theorem 1.1. ([1], Lemma 4.1) Let $\xi \in \mathbb{R}$. Suppose that the numbers $\alpha, \beta, \gamma$, $C_{1}, C_{2}, C_{3} \in \mathbb{R}^{+}$satisfy $\alpha \leq \beta$ and $\gamma \geq 1$ and there exist a sequence $\frac{p_{n}}{q_{n}} \in \mathbb{Q}$ such that for every $n$

$$
\begin{gathered}
q_{n}<q_{n+1} \leq C_{1} q_{n}^{\gamma}, \\
\frac{C_{2}}{q_{n}^{1+\beta}} \leq\left|\xi-\frac{p_{n}}{q_{n}}\right| \leq \frac{C_{3}}{q_{n}^{1+\alpha}} .
\end{gathered}
$$

Then, the irrationality measure is

$M S C$ (2010): primary 11J82.

Keywords: irrationality measure, infinite series.

This work was supported by the Grant P201/12/2351 of the Czech Science Foundation. 


$$
\mu(\xi) \leq \frac{(1+\beta) \gamma}{\alpha}
$$

Hančl and Filip [3] proved the following theorem.

Theorem 1.2. ([3], Theorem 2) Suppose that the numbers $\varepsilon, R, S \in \mathbb{R}^{+}$satisfy $S<\frac{\varepsilon}{1+\varepsilon}$ and $R>\frac{1}{1-S}$. Let $a_{n}, b_{n} \in \mathbb{N}$ be two sequences with $a_{n}$ nondecreasing such that

$$
\begin{gathered}
\limsup _{n \rightarrow \infty} a_{n}^{\frac{1}{(R+1)^{n}}}>1, \\
b_{n}=O\left(a_{n}^{S}\right)
\end{gathered}
$$

and, for every sufficiently large positive integer $n$,

$$
a_{n}>n^{1+\varepsilon} .
$$

Then, the number $\xi:=\sum_{n=1}^{\infty} \frac{b_{n}}{a_{n}}$ is irrational and has irrationality measure

$$
\mu(\xi) \geq \max \{2,(1-S) R\} .
$$

Some other results on irrationality measure of infinite series can be found in [2]. For a survey on irrationality measure and other topics of transcendental number theory, see [4].

This paper presents new upper bounds for irrationality measure of infinite series of rational numbers. Our results depend only on the speed of convergence of the series and do not depend on the arithmetical properties of the terms.

\section{Results}

Theorem 2.1. Let the numbers $E, F, G, S, U, V \in \mathbb{R}$ satisfy $1<E \leq F<$ $E^{(1-S) U}, 0 \leq S<1 \leq G$ and $1<U \leq V$. Let $T_{n} \in \mathbb{R}^{+}$be a sequence of numbers and, for every $n \in \mathbb{N}$, put $H_{n}:=\sum_{k=1}^{n} T_{k}$. Suppose that the following relations hold.

$$
\begin{gathered}
U=\liminf _{n \rightarrow \infty} \frac{T_{n+1}}{H_{n}} \leq \limsup _{n \rightarrow \infty} \frac{T_{n+1}}{H_{n}}=V, \\
\limsup _{n \rightarrow \infty} \frac{H_{n+1}}{H_{n}}=G .
\end{gathered}
$$

Let $a_{n}, b_{n} \in \mathbb{N}$ be sequences with $a_{n}$ nondecreasing such that

$$
\begin{gathered}
E=\liminf _{n \rightarrow \infty} a_{n}^{\frac{1}{T_{n}}} \leq \limsup _{n \rightarrow \infty} a_{n}^{\frac{1}{T_{n}}}=F, \\
\limsup _{n \rightarrow \infty} \frac{\log b_{n}}{\log a_{n}}=S .
\end{gathered}
$$

Then, the number $\xi:=\sum_{n=1}^{\infty} \frac{b_{n}}{a_{n}}$ has irrationality measure

$$
\mu(\xi) \leq \frac{\left(\frac{\log F}{\log E}\right)^{2} V G}{\frac{\log E}{\log F}(1-S) U-1} .
$$


In the case of $a_{n} \mid a_{n+1}$, we obtain a better result.

Theorem 2.2. Let the numbers $E, F, S, U, V \in \mathbb{R}$ satisfy $0 \leq S<1<U \leq V$ and $1<E \leq F<E^{(1-S) U}$. Let $T_{n} \in \mathbb{R}^{+}$be a sequence of numbers such that

$$
U=\liminf _{n \rightarrow \infty} \frac{T_{n+1}}{T_{n}} \leq \limsup _{n \rightarrow \infty} \frac{T_{n+1}}{T_{n}}=V .
$$

Let $a_{n}, b_{n} \in \mathbb{N}$ be sequences such that $a_{n} \mid a_{n+1}$ for every $n$ and that

$$
\begin{gathered}
E=\liminf _{n \rightarrow \infty} a_{n}^{\frac{1}{T_{n}}} \leq \limsup _{n \rightarrow \infty} a_{n}^{\frac{1}{T_{n}}}=F, \\
\limsup _{n \rightarrow \infty} \frac{\log b_{n}}{\log a_{n}}=S .
\end{gathered}
$$

Then, the number $\xi:=\sum_{n=1}^{\infty} \frac{b_{n}}{a_{n}}$ has irrationality measure

$$
\mu(\xi) \leq \frac{\left(\frac{\log F}{\log E}\right)^{2} V^{2}}{\frac{\log E}{\log F}(1-S) U-1} .
$$

We obtain the results more easily if the sequence $T_{n}$ is geometric.

Corollary 2.3. Let the numbers $E, F, S, T \in \mathbb{R}$ satisfy $T>2,1<E \leq F<$ $E^{(1-S)(T-1)}$ and $0 \leq S<1$. Let $a_{n}, b_{n} \in \mathbb{N}$ be sequences with $a_{n}$ nondecreasing such that

$$
\begin{gathered}
E=\liminf _{n \rightarrow \infty} a_{n}^{\frac{1}{T^{n}}} \leq \limsup _{n \rightarrow \infty} a_{n}^{\frac{1}{T^{n}}}=F, \\
\limsup _{n \rightarrow \infty} \frac{\log b_{n}}{\log a_{n}}=S .
\end{gathered}
$$

Then the number $\xi:=\sum_{n=1}^{\infty} \frac{b_{n}}{a_{n}}$ has irrationality measure

$$
\mu(\xi) \leq \frac{\left(\frac{\log F}{\log E}\right)^{2}(T-1) T}{\frac{\log E}{\log F}(1-S)(T-1)-1} .
$$

Corollary 2.4. Let the numbers $E, F, S, T \in \mathbb{R}$ satisfy $0 \leq S<1<T$ and $1<E \leq F<E^{(1-S) T}$. Let $a_{n}, b_{n} \in \mathbb{N}$ be sequences such that $a_{n} \mid a_{n+1}$ for every $n$ and that

$$
\begin{gathered}
E=\liminf _{n \rightarrow \infty} a_{n}^{\frac{1}{T^{n}}} \leq \limsup _{n \rightarrow \infty} a_{n}^{\frac{1}{T^{n}}}=F, \\
\limsup _{n \rightarrow \infty} \frac{\log b_{n}}{\log a_{n}}=S .
\end{gathered}
$$

Then, the number $\xi:=\sum_{n=1}^{\infty} \frac{b_{n}}{a_{n}}$ has irrationality measure

$$
\mu(\xi) \leq \frac{\left(\frac{\log F}{\log E}\right)^{2} T^{2}}{\frac{\log E}{\log F}(1-S) T-1} .
$$


Example 2.5. For every $n \in \mathbb{N}$ put

$$
x_{n}= \begin{cases}n^{2} & \text { if } n \text { is a prime, } \\ n & \text { if } n \text { is not a prime. }\end{cases}
$$

Using Theorem 2.1 with $a_{n}=x_{n}^{4^{n}}, b_{n}=n !, T_{n}=4^{n} \log _{2} n, E=2, F=4, G=4$, $S=0, U=V=3$, we obtain

$$
\mu\left(\sum_{n=1}^{\infty} \frac{n !}{x_{n}^{4^{n}}}\right) \leq 96 .
$$

Example 2.6. Let $A>1$ be a real number. Using Theorem 2.2 with $a_{n}=$ $n !^{\left\lfloor A^{n}\right\rfloor}, b_{n}=1, T_{n}=\left\lfloor A^{n}\right\rfloor\left(n \ln n-n+\frac{1}{2} \ln n\right), E=F=\mathrm{e}, S=0, U=V=A$, together with Stirling's formula, we obtain

$$
\mu\left(\sum_{n=1}^{\infty} \frac{1}{n !\left\lfloor A^{n}\right\rfloor}\right) \leq \frac{A^{2}}{A-1} .
$$

Example 2.7. Let $A, B$ be real numbers with $A, B>2$. Then, Theorem 1.2 and Corollary 2.3 imply that

$$
B-1 \leq \mu\left(\sum_{n=1}^{\infty} \frac{1}{\left\lfloor A^{\left.B^{n}\right\rfloor}\right.}\right) \leq \frac{(B-1) B}{B-2} .
$$

Remark 2.8. Our results and proofs contain logarithms, but they do not depend on the base of the logarithms.

\section{Proofs}

We will modify Theorem 1.1 a little.

Lemma 3.1. Let $\xi \in \mathbb{R}$. Suppose that numbers $\alpha, \beta, \gamma, C_{4}, C_{5}, C_{6} \in \mathbb{R}^{+}$and $N_{1} \in \mathbb{N}$ satisfy $1<\alpha \leq \beta$ and $\gamma \geq 1$ and there exist sequences $p_{n} \in \mathbb{Z}$ and $q_{n} \in \mathbb{N}$ with $\lim _{n \rightarrow \infty} q_{n}=\infty$ such that for every $n \geq N_{1}$

$$
\begin{gathered}
q_{n} \leq q_{n+1} \leq C_{4} q_{n}^{\gamma}, \\
\frac{C_{5}}{q_{n}^{\beta}} \leq\left|\xi-\frac{p_{n}}{q_{n}}\right| \leq \frac{C_{6}}{q_{n}^{\alpha}} .
\end{gathered}
$$

Then, the irrationality measure is

$$
\mu(\xi) \leq \frac{\beta \gamma}{\alpha-1}
$$

Proof. The proof is the same as that of Lemma 4.1 in [1], only the constants $\alpha, \beta$ are shifted by one. Lemma 4.1 in [1] uses the strict inequality $q_{n}<q_{n+1}$ only to ensure that $\lim _{n \rightarrow \infty} q_{n}=\infty$, so we use the latter in the assumption of our Lemma 3.1. 
In the following proofs the constants $C_{i}>0$ and $N_{i} \in \mathbb{N}$ depend on $\delta$ and do not depend on $n$.

Proof. (Theorem 2.1) Let $\delta \in\left(0, \min \left\{E-1, \frac{1-S}{3}, U-1\right\}\right)$ be so small that

$$
F+\delta<(E-\delta)^{(1-S-3 \delta)(U-\delta)} .
$$

Equations (2.1), (2.2), (2.3) and (2.4) imply that there exists $N_{2} \in \mathbb{N}$ such that, for every $n \geq N_{2}$,

$$
\begin{gathered}
U-\delta<\frac{T_{n+1}}{H_{n}}<V+\delta, \\
\frac{H_{n+1}}{H_{n}}<G+\delta, \\
(E-\delta)^{T_{n}}<a_{n}<(F+\delta)^{T_{n}}, \\
b_{n}<a_{n}^{S+\delta} .
\end{gathered}
$$

From (3.1), we obtain for every $n \geq N_{2}$

$$
\frac{H_{n+1}}{H_{n}}=\frac{H_{n}+T_{n+1}}{H_{n}}>1+U-\delta>2
$$

and

$$
H_{n} \geq(1+U-\delta)^{n-N_{2}}
$$

Using (3.1) again, we obtain for every $n \geq N_{2}+1$

$$
T_{n}>(U-\delta) H_{n-1}>(U-\delta)(1+U-\delta)^{n-N_{2}-1}=C_{7}(1+U-\delta)^{n},
$$

where $C_{7}=\frac{U-\delta}{(1+U-\delta)^{N_{2}+1}}$. Therefore, there exists $N_{3}>N_{2}$ such that, for every $n \geq N_{3}$,

$$
a_{n}>(E-\delta)^{C_{7}(1+U-\delta)^{n}}>2^{n} .
$$

In particular, $\lim _{n \rightarrow \infty} a_{n}=\infty$. Let $N_{4} \geq N_{3}$ be so large that, for every $n \geq N_{4}$,

$$
\begin{gathered}
\left\lceil\log _{2} a_{n}\right\rceil<a_{n}^{\delta}, \\
a_{n}^{\delta}+\frac{1}{2^{1-S-\delta}-1}<a_{n}^{2 \delta} .
\end{gathered}
$$

Put $q_{n}:=\prod_{k=1}^{n} a_{k}$. Then, there exists a sequence $p_{n}$ of positive integers such that, for every $n \in \mathbb{N}$,

$$
\sum_{k=1}^{n} \frac{b_{k}}{a_{k}}=\frac{p_{n}}{q_{n}} .
$$

Equation (3.3) implies that, for every $n \geq N_{4}$,

$$
\begin{aligned}
q_{n} & =q_{N_{4}-1} \prod_{k=N_{4}}^{n} a_{k}>q_{N_{4}-1} \prod_{k=N_{4}}^{n}(E-\delta)^{T_{k}} \\
& =q_{N_{4}-1}(E-\delta)^{H_{n}-H_{N_{4}-1}}=C_{8}(E-\delta)^{H_{n}},
\end{aligned}
$$

where $C_{8}=\frac{q_{N_{4}-1}}{(E-\delta)^{H_{N_{4}-1}}}$. Similarly, 


$$
q_{n}<q_{N_{4}-1} \prod_{k=N_{4}}^{n}(F+\delta)^{T_{k}}=q_{N_{4}-1}(F+\delta)^{H_{n}-H_{N_{4}-1}}=C_{9}(F+\delta)^{H_{n}},
$$

where $C_{9}=\frac{q_{N_{4}-1}}{(F+\delta)^{H_{N_{4}-1}}}$.

Put $\alpha:=\frac{\log (E-\delta)}{\log (F+\delta)}(1-S-3 \delta)(U-\delta)>1$. Equation (3.4) implies that, for every $n \geq N_{4}$,

$$
\begin{aligned}
\left|\xi-\frac{p_{n}}{q_{n}}\right| & =\sum_{k=n+1}^{\infty} \frac{b_{k}}{a_{k}}<\sum_{k=n+1}^{\infty} \frac{1}{a_{k}^{1-S-\delta}} \\
& =\sum_{k=n+1}^{\left\lceil\log _{2} a_{n+1}\right\rceil} \frac{1}{a_{k}^{1-S-\delta}}+\sum_{k=\left\lceil\log _{2} a_{n+1}\right\rceil+1}^{\infty} \frac{1}{a_{k}^{1-S-\delta}} .
\end{aligned}
$$

For the first summand, we obtain from the monotonicity of $a_{n}$ and from (3.6) that

$$
\sum_{k=n+1}^{\left\lceil\log _{2} a_{n+1}\right\rceil} \frac{1}{a_{k}^{1-S-\delta}} \leq \frac{\left\lceil\log _{2} a_{n+1}\right\rceil}{a_{n+1}^{1-S-\delta}}<\frac{1}{a_{n+1}^{1-S-2 \delta}} .
$$

Equation (3.5) implies for the second summand that

$$
\begin{aligned}
\sum_{k=\left\lceil\log _{2} a_{n+1}\right\rceil+1}^{\infty} \frac{1}{a_{k}^{1-S-\delta}} & <\sum_{k=\left\lceil\log _{2} a_{n+1}\right\rceil+1}^{\infty} \frac{1}{2^{(1-S-\delta) k}}=\frac{C_{10}}{2^{(1-S-\delta)\left\lceil\log _{2} a_{n+1}\right\rceil}} \\
& \leq \frac{C_{10}}{a_{n+1}^{1-S-\delta}},
\end{aligned}
$$

where $C_{10}=\frac{1}{2^{1-S-\delta}-1}$. This, (3.10), (3.7), (3.3), (3.1) and (3.9) imply

$$
\begin{aligned}
\left|\xi-\frac{p_{n}}{q_{n}}\right| & <\frac{1}{a_{n+1}^{1-S-2 \delta}}+\frac{C_{10}}{a_{n+1}^{1-S-\delta}}<\frac{1}{a_{n+1}^{1-S-3 \delta}}<\frac{1}{(E-\delta)^{T_{n+1}(1-S-3 \delta)}} \\
& =\frac{1}{(F+\delta)^{\frac{\log (E-\delta)}{\log (F+\delta)}(1-S-3 \delta) \frac{T_{n+1}}{H_{n}} H_{n}}}<\frac{1}{(F+\delta)^{\alpha H_{n}}}<\frac{C_{11}}{q_{n}^{\alpha}},
\end{aligned}
$$

where $C_{11}=C_{9}^{\alpha}$. This in particular implies that the series $\xi=\sum_{n=1}^{\infty} \frac{b_{n}}{a_{n}}$ converges.

Put $\beta:=\frac{\log (F+\delta)}{\log (E-\delta)}(V+\delta)>\alpha$. Then, (3.3), (3.1) and (3.8) imply for every $n \geq N_{4}$ that

$$
\begin{aligned}
\left|\xi-\frac{p_{n}}{q_{n}}\right| & =\sum_{k=n+1}^{\infty} \frac{b_{n}}{a_{n}}>\frac{1}{a_{n+1}}>\frac{1}{(F+\delta)^{T_{n+1}}}=\frac{1}{(E-\delta)^{\frac{\log (F+\delta)}{\log (E-\delta)} \frac{T_{n+1}}{H_{n}} H_{n}}} \\
& >\frac{1}{(E-\delta)^{\beta H_{n}}}>\frac{C_{12}}{q_{n}^{\beta}}
\end{aligned}
$$


where $C_{12}=C_{8}^{\beta}$.

Put $\gamma:=\frac{\log (F+\delta)}{\log (E-\delta)}(G+\delta)>1$. Then, (3.9), (3.2) and (3.8) imply for every $n \geq N_{4}$ that

$$
\begin{aligned}
q_{n} & \leq q_{n+1}<C_{9}(F+\delta)^{H_{n+1}}=C_{9}(E-\delta)^{\frac{\log (F+\delta)}{\log (E-\delta)} \frac{H_{n+1}}{H_{n}} H_{n}} \\
& <C_{9}(E-\delta)^{\gamma H_{n}}<C_{13} q_{n}^{\gamma},
\end{aligned}
$$

where $C_{13}=\frac{C_{9}}{C_{8}^{\gamma}}$.

Equations (3.11), (3.12), (3.13) with Lemma 3.1 imply that

$$
\mu(\xi) \leq \frac{\beta \gamma}{\alpha-1}=\frac{\left(\frac{\log (F+\delta)}{\log (E-\delta)}\right)^{2}(V+\delta)(G+\delta)}{\frac{\log (E-\delta)}{\log (F+\delta)}(1-S-3 \delta)(U-\delta)-1} .
$$

The proof of Theorem 2.1 is finished by letting $\delta \rightarrow 0$.

Proof. (Theorem 2.2) Let $\delta \in\left(0, \min \left\{E-1, \frac{1-S}{3}, U-1\right\}\right)$ be so small that

$$
F+\delta<(E-\delta)^{(1-S-3 \delta)(U-\delta)} .
$$

Equations (2.5), (2.6) and (2.7) imply that there exists $N_{5} \in \mathbb{N}$ such that for every $n \geq N_{5}$

$$
\begin{gathered}
U-\delta<\frac{T_{n+1}}{T_{n}}<V+\delta \\
(E-\delta)^{T_{n}}<a_{n}<(F+\delta)^{T_{n}}, \\
b_{n}<a_{n}^{S-\delta}
\end{gathered}
$$

From (3.14), we obtain for every $n \geq N_{5}$ that

$$
T_{n}>T_{N_{5}}(U-\delta)^{n-N_{5}}=C_{14}(U-\delta)^{n},
$$

where $C_{14}=\frac{T_{N_{5}}}{(U-\delta)^{N_{5}}}$. This with (3.15) implies that there exists $N_{6} \geq N_{5}$ such that for every $n \geq N_{6}$

$$
a_{n}>(E-\delta)^{C_{14}(U-\delta)^{n}}>2^{n}
$$

In particular, $\lim _{n \rightarrow \infty} a_{n}=\infty$.

Let $N_{7} \geq N_{6}$ be so large a positive integer that, for every $n \geq N_{7}$, the inequalities (3.6) and (3.7) hold.

For every $n \in \mathbb{N}$, put $q_{n}:=a_{n}$. From the property $a_{n} \mid a_{n+1}$ we obtain that there exists a sequence $p_{n}$ of positive integers such that, for every $n \in \mathbb{N}$,

$$
\sum_{k=1}^{n} \frac{b_{k}}{a_{k}}=\frac{p_{n}}{q_{n}}
$$



have

Put $\alpha:=\frac{\log (E-\delta)}{\log (F+\delta)}(1-S-3 \delta)(U-\delta)>1$. Then, from (3.15) and (3.14), we

$$
a_{n+1}>(E-\delta)^{T_{n+1}}=(F+\delta)^{T_{n} \frac{\log (E-\delta)}{\log (F+\delta)} \frac{T_{n+1}}{T_{n}}}>a_{n}^{\frac{\log (E-\delta)}{\log (F+\delta)} \frac{T_{n+1}}{T_{n}}}>a_{n}^{\frac{\alpha}{1-S-3 \delta}} .
$$

Now, for every $n \geq N_{7}$, we will find an upper bound for the error of approximation of $\xi$. As in the proof of Theorem 2.1, we obtain

$$
\left|\xi-\frac{p_{n}}{q_{n}}\right|<\frac{1}{a_{n+1}^{1-S-3 \delta}}
$$

with the series $\xi=\sum_{n=1}^{\infty} \frac{b_{n}}{a_{n}}$ converging. Equation (3.16) then implies

$$
\left|\xi-\frac{p_{n}}{q_{n}}\right|<\frac{1}{a_{n+1}^{1-S-3 \delta}}<\frac{1}{a_{n}^{\alpha}}=\frac{1}{q_{n}^{\alpha}} .
$$

Put $\beta:=\gamma:=\frac{\log (F+\delta)}{\log (E-\delta)}(V+\delta)>\alpha>1$. Equations (3.15) and (3.14) imply that, for every $n \geq N_{7}$,

$$
\begin{aligned}
q_{n} & \leq q_{n+1}=a_{n+1}<(F+\delta)^{T_{n+1}}=(E-\delta)^{T_{n} \frac{\log (F+\delta)}{\log (E-\delta)} \frac{T_{n+1}}{T_{n}}} \\
& <a_{n}^{\frac{\log (F+\delta)}{\log (E-\delta)} \frac{T_{n+1}}{T_{n}}}<a_{n}^{\gamma}=q_{n}^{\gamma} .
\end{aligned}
$$

From this, we obtain a lower bound for the error of approximation of $\xi$

$$
\left|\xi-\frac{p_{n}}{q_{n}}\right|=\sum_{k=n+1}^{\infty} \frac{b_{k}}{a_{k}}>\frac{1}{a_{n+1}}>\frac{1}{a_{n}^{\gamma}}=\frac{1}{q_{n}^{\beta}} .
$$

Equations (3.17), (3.18), (3.19) with Lemma 3.1 imply that

$$
\mu(\xi) \leq \frac{\beta \gamma}{\alpha-1}=\frac{\left(\frac{\log (F+\delta)}{\log (E-\delta)}\right)^{2}(V+\delta)^{2}}{\frac{\log (E-\delta)}{\log (F+\delta)}(1-S-3 \delta)(U-\delta)} .
$$

The proof of Theorem 2.2 is finished by letting $\delta \rightarrow 0$.

Proof. (Corollary 2.3) Put $T_{n}=T^{n}, G=T, U=V=T-1$ and use Theorem 2.1.

Proof. (Corollary 2.4) Put $T_{n}=T^{n}, U=V=T$ and use Theorem 2.2.

\section{REFERENCES}

[1] B. Adamczewski and T. Rivoal, Irrationality measures for some automatic real numbers, Math. Proc. Camb. Philos. Soc. 147 (2009), 659-678.

[2] D. Duverney, Irrationality of fast converging series of rational numbers, J. Math. Sci., Tokyo 8 (2001), 275-316.

[3] J. Hančl and F. Filip, Irrationality measure of sequences, Hiroshima Math. J. 35 (2005), 183-195.

[4] A. N. Parshin and I. R. Shafarevich (eds.), Number Theory IV. Transcendental Numbers, Springer-Verlag, Berlin, Heidelberg, 1998.

[5] H. Davenport, K.F. Roth, Rational approximations to algebraic numbers, Mathematika 2 (1955), 160-167. 
[6] J. Sondow, Irrationality measures, irrationality bases, and a theorem of Jarnik, Proceedings of Journées Arithmétiques, Graz 2003, arXiv:math/0406300.

Jan Šustek, Department of Mathematics, Faculty of Science, University of Ostrava, 30. dubna 22, Ostrava, 701 03, Czech Republic e-mail: jan.sustek@osu.cz 
